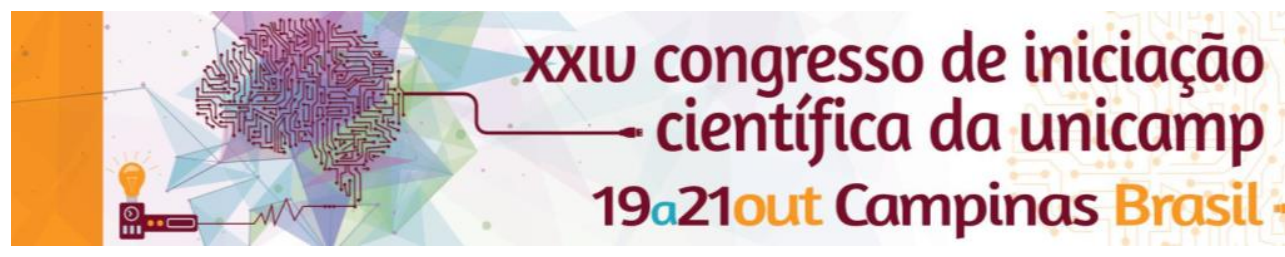

\title{
Synthesis and characterization of segmented polyurethanes
}

\author{
André Sanches Bevilacqua*, Rafael Bergamo Trinca, Maria Isabel Felisberti.
}

\begin{abstract}
Segmented polyurethanes (SPUs) based on PEG, PTMC and PLLA as macrodiols, HDI as diisocyanate and BDO as chain extender were synthetized and characterized with respect to composition, thermal and dynamical mechanical properties. The SPUs are heterogeneous and the thermal events observed in DSC and DMA analyses are related to the macrodiols components, being affected by the global composition.
\end{abstract}

\section{Key words:}

Ternary,segmented polyurethanes, multicomponent.

\section{Introduction}

Segmented polyurethanes (SPUs) are a class of polyurethanes constituted by blocks such as polyester and polyether macrodiols. Combination of different macrodiols results in polyurethanes with different properties ${ }^{1}$. In this work, ternary SPUs were synthetized based on poly(Ilactide) (PLLA, a semicrystalline and hidrophobic polyester), poly(ethylene glycol) (PEG, a semicrystalline and hydrophilic poliether), and poly(trimethylene carbonate) (PTMC, an amorphous and hydrophobic policarbonate) as macrodiols, 1,6 hexanediisocyanate (HDI) and 1,4 butanediol (BDO) as chain extender. The combination of these macrodiols in different amounts allows the control of hydrophillic-hydrophobic balance and the thermal and mechanical properties of the SPU ${ }^{2}$.

\section{Results and Discussion}

The SPUs were synthetized using PLLA, PTMC and PEG macrodiols with molar mass around $2 \mathrm{kDa}$ and polydispersity around 1.7-2.0. The nomenclature of the ternary SPU is based on the mass ratio of PEG, PLLA and PTMC. For example, the SPU 111 was synthetized from a solution containing PEG:PLLA:PTMC mass ratio of 1:1:1. In general, the compositions of SPUs, determined from RMN data, are close to that of the macrodiols mass ratio in the reaction medium, as can be observed in Figure 1. The exception is SPU211, which is poorer in PEG compared with the planned composition.

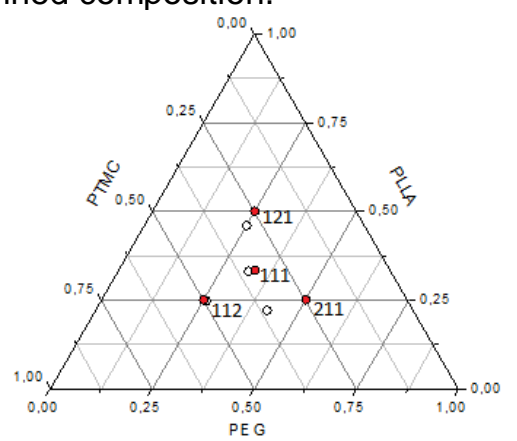

Figure 1 - PEG:PLLA:PTMC mass ratio in SPU (०) and in the reaction medium $(\bullet)$.

Differential Scanning Calorimetry (DSC) and dynamic mechanical analysis (DMA) were used to determine the glass transition (Tg), crystallization (Tc) and melting (Tm) temperatures (Figure 2). DSC curves show multiple events dependent on SPU compositions: Tg, PEG crystallization and melting (10 and $32{ }^{\circ} \mathrm{C}$ respectively) and PLLA crystallization and melting (38 and $77^{\circ} \mathrm{C}$, respectively).
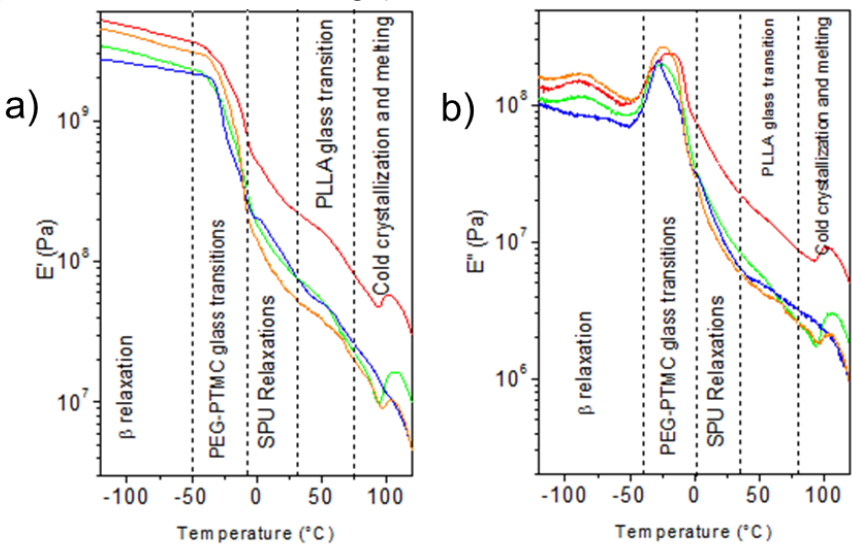

c)

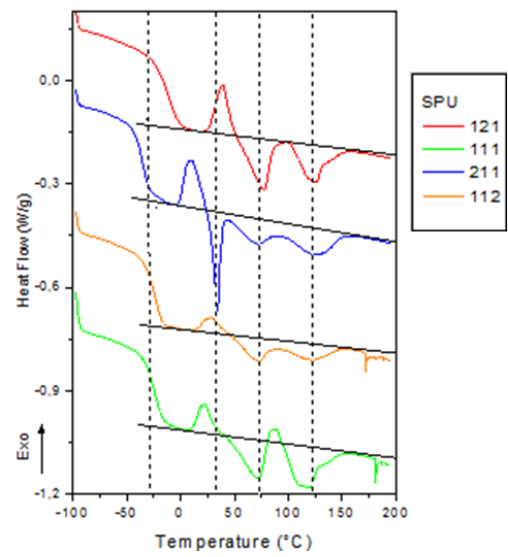

Figure 2- a) Storage and b) loss moduli as a function of temperature. c) DSC curves $2^{\text {nd }}$ heating scan.

DMA curves show events which can be associated to: $\beta$ transitions, PEG-PTMC glass transitions, PLLA glass transitions, cold crystallization and melting. DSC and DMA data indicate that all SPUs are heterogenous and multiphase materials.

\section{Conclusions}

SPUs are heterogeneous polymers presenting transitions related to each compound. Varying the composition allows designing the properties of the polyurethanes.

\section{Acknowledgement}

CNPq (444392/2014-9) and Fapesp (2010/17804-7 and 2015/25406-5). 